\title{
Lumen
}

Selected Proceedings from the Canadian Society for Eighteenth-Century Studies

\section{La Lettre à d'Alembert sur les spectacles, ou l'inscription européenne du républicanisme}

\section{Lucien Nouis}

Volume 30, 2011

URI : https://id.erudit.org/iderudit/1007718ar

DOI : https://doi.org/10.7202/1007718ar

Aller au sommaire du numéro

Éditeur(s)

Canadian Society for Eighteenth-Century Studies / Société canadienne d'étude du dix-huitième siècle

ISSN

1209-3696 (imprimé)

1927-8284 (numérique)

Découvrir la revue

Citer cet article

Nouis, L. (2011). La Lettre à d'Alembert sur les spectacles, ou l'inscription européenne du républicanisme. Lumen, 30, 99-112.

https://doi.org/10.7202/1007718ar

Copyright (c) Canadian Society for Eighteenth-Century Studies / Sociéte canadienne d'étude du dix-huitième siècle, 2011
Ce document est protégé par la loi sur le droit d'auteur. L'utilisation des services d'Érudit (y compris la reproduction) est assujettie à sa politique d'utilisation que vous pouvez consulter en ligne.

https://apropos.erudit.org/fr/usagers/politique-dutilisation/ 


\section{La Lettre à d'Alembert sur les spectacles, ou l'inscription européenne du républicanisme}

La Lettre à d'Alembert sur les spectacles, longtemps considérée comme une production marginale dans l'œuvre de Rousseau, voire réactionnaire, a retrouvé depuis maintenant une trentaine d'années la place qui lui revient ${ }^{1}$. Sans doute, le fait que son propos se situe aux confins de l'esthétique et de la politique n'est pas étranger à une telle redécouverte : Rousseau y traite du théâtre, certes, mais sur l'horizon d'une interrogation beaucoup plus large sur la stabilité du républicanisme, sur ses frontières et ses limites, et par là sur l'identité d'un peuple. La thèse qui sous-tend le mouvement rhétorique de la Lettre est en effet celle que l'on connaît - toute forme de gouvernement, et en particulier le gouvernement républicain, repose sur une manière d'être, sur un ethos des citoyens : «Il n'y a point d'État bien constitué où l'on ne trouve des usages qui tiennent à la forme du gouvernement et servent à la maintenir ${ }^{2} . »$ Or, parce qu'il existe une telle identité entre la partie (les citoyens) et le tout qui les rassemble (la république), la moindre défaillance dans les mœurs, le plus léger dérèglement moral, deviennent annonciateurs de ruine. Dans le cas de Genève, on le sait, c'est le théâtre qui constitue cette menace et qui fait que la petite république risque de devenir une autre Sparte ou Rome : «Il ne faut point le dissimuler ; les intentions sont droites encore ; mais les mœurs inclinent déjà visiblement vers la décadence, et nous suivons de loin les traces des mêmes

1 Pour un jalon important dans ce renouveau d'intérêt critique pour ce texte, voir : Patrick Coleman, Rousseau's Political Imagination. Rule and Representation in the Lettre à d'Alembert, Genève, Droz, 1984.

2 Jean-Jacques Rousseau, Lettre à d'Alembert in CEuvres complètes, Paris, Gallimard, collection «Bibliothèque de la Pléiade», 1962, vol. V, p. 90. Toutes les références suivantes renvoient à cette édition. 
peuples dont nous ne laissons pas de craindre le sort ${ }^{3}$.» Rousseau lance donc avec la Lettre un appel au retour vers une citoyenneté maintenue de l'intérieur contre tous les périls dont le théâtre est la métonymie : pour la conservation du système politique, il faudrait trouver les moyens de préserver les mœurs des citoyens de toute influence délétère, quitte à enfermer la ville derrière ses montagnes et ses murs.

On reconnaît ici un lieu commun très présent dans la pensée politique du dix-huitième siècle. Les républiques - et il faudrait entendre par là les gouvernements fondés sur l'autorité de la loi et l'égalité des citoyens - seraient marquées en leur sein par une faiblesse systémique les prédisposant à l'effondrement politique. Montesquieu va tout à fait dans le sens de cette idée lorsqu'il décrit dans l'Esprit des lois la difficulté qu'il y a à maintenir une égalité mesurée, ni excessive ni déficiente, à l'intérieur du démos : «La démocratie a [...] deux excès à éviter : l'esprit d'inégalité, qui mène à l'aristocratie, ou au gouvernement d'un seul ; et l'esprit d'égalité extrême, qui la conduit au despotisme d'un seul, comme le despotisme d'un seul finit par la conquête ${ }^{4}$.» Prise entre le trop peu d'égalité et le trop plein d'égalité, ne se maintenant que difficilement dans une sorte de médiocrité positive, la république ou démocratie est ici constamment tirée vers le gouvernement d'un seul, soit monarchique, soit despotique. L'autre principal facteur de fragilité mentionné par Montesquieu à propos des républiques, lié au premier et bien connu lui aussi, tient au fait que leur moteur est la «vertu»: c'est le sacrifice de chacun à la cause partagée, l'exaltation de la communauté, qui assure sa cohésion à la structure sociopolitique. À la différence d'autres systèmes, tels ceux du despotisme, dont le principe est la peur, ou de la monarchie, qui vit de l'impératif égoïste du désir d'honneurs, la survie du républicanisme dépend donc en dernier

3 Jean-Jacques Rousseau, Lettre à d'Alembert, p. 120.

4 Montesquieu, De l'esprit des lois, éd. Victor Goldschmidt, Paris, GF Flammarion, 1979 , p. 244. L'idée de la fragilité des systèmes démocratiques a comme on le sait une très longue histoire, que l'on peut faire remonter à Platon et Aristote. On pourra consulter sur ce sujet un texte classique, qui montre comment au cœur de toute tentative républicaine se pose la question de la stabilité et de la pérennité du système : J. G. A. Pocock, The Machiavellian Moment. Florentine Political Thought and the Atlantic Republican Tradition, Princeton et Oxford, Princeton University Press, 1975. Cette question reste d'ailleurs plus que jamais d'actualité — parmi les textes récents et marquants qui en traitent, on pourra retenir en premier lieu Voyou, où Jacques Derrida renouvelle et complexifie le lieu commun en ayant recours à la métaphore des troubles auto-immunitaires : Jacques Derrida, Voyous, Paris, Galilée, 2003. 
lieu de l'assujettissement entier et volontaire de chacun à l'intérêt du groupe. Qu'une intrusion vienne à menacer cette homogénéité, qu'une altérité fasse interférence, de manière réelle ou fantasmée, et c'est tout le corps social qui est mis en péril.

Mais au lieu de l'attitude de détachement analytique adoptée par Montesquieu (certes bien compréhensible dans la mesure où ce dernier n'est pas républicain mais partisan d'une monarchie parlementaire modérée), on retrouve chez Rousseau, dès la préface, une forme de pathos, une dimension émotionnelle très forte, qui signale à quel point sa défense d'un républicanisme assiégé est investie par l'autobiographie ${ }^{5}$. Le philosophe ne peut en effet écrire Genève en effaçant l'histoire particulièrement problématique de sa propre citoyenneté ; le récit des maux de la république se fait chez lui sur le palimpseste du récit des maux du citoyen. C'est pourquoi il semble fondé de dire que s'il y a chez Rousseau une obsession de l'autonomie, de l'insularité, ainsi qu'une peur correspondante de la contagion, ce n'est pas seulement parce qu'il propose - comme cela est souvent rappelé - une nouvelle conception du sujet social aliéné, mais bien aussi parce que beaucoup de ses écrits ont pour horizon une réflexion sur la perte de la citoyenneté et sur le déclin des républiques. La Lettre pourrait ainsi se lire en partie comme le versant politique de la question de l'autonomie perdue et de l'espace intime fracturé. Or, au-delà de tout ce qui mériterait d'être rappelé à propos de ces effets de circulation entre autobiographie et pensée politique, cette indéniable complexité rhétorique fait que la république devient ici plus encore qu'ailleurs une chose écrite, un texte appuyé d'une signature.

C'est d'ailleurs lorsqu'elle est envisagée sous cet angle que la Lettre qui après tout contient une critique du théâtre qui n'est pas sans poser problème aux modernes que nous sommes - se révèle dans toute son originalité : cette double mise en récit du républicanisme (les maux de Rousseau devenant ceux de Genève, dans un passage constant du «je» au «nous») permet non seulement au philosophe de dépasser le schème

5 Au-delà de cette différence de ton, Ourida Mostefai a cependant tout à fait raison d'aller dans le sens d'un rapprochement entre ces deux penseurs dans sa lecture de la Lettre à d'Alembert : «[...] Rousseau s'inspire de l'auteur de l'Esprit des lois lorsqu'il décide d'examiner l'institution du théâtre par rapport à la constitution de l'État, c'est à dire de considérer le théâtre non en lui-même, mais par rapport au corps politique, et comme faisant partie de la législation.» (Ourida Mostefai, Le Citoyen de Genève et la république des lettres. Étude sur la controverse autour de la Lettre à d'Alembert de Jean-Jacques Rousseau, New York, Peter Lang, collection «The Age of Revolution and Romanticism", 2003, p. 5.) 
éculé des méfaits du théâtre, mais aussi de se dégager du lieu commun de la fragilité des républiques. On s'aperçoit en effet très vite que l'appel à la défense de l'ethos républicain menacé passe chez Rousseau par une réflexion subtile sur la façon dont s'écrit la république. Au cœur de la Lettre, comme une sorte de point limite de l'effectivité de l'écriture en politique, il y aurait l'idée que protéger la république, ce serait aussi en préserver l'image textuelle, en garder les frontières à l'intérieur d'un espace public virtuel, espace que l'on pourrait définir comme étant celui d'une pensée politique européenne dont l'élaboration passerait justement par toutes les formes de l'écrit et en particulier par l'immense projet éditorial que constitue l'Encyclopédie. On sait combien l'écriture est chez Rousseau indissociable d'une identification avec la citoyenneté républicaine : il signe ses textes en tant que citoyen, pour le bien public, dans l'effacement vertueux - c'est le sens de sa fameuse devise vitam impendere vero rappelé dans une note célèbre de la Lettre ${ }^{6}$. Mais l'écriture «républicaine» ou «citoyenne» est ici à envisager différemment, c'est-àdire plus seulement comme l'acte héroïque de celui qui défend la vérité au péril de sa vie, mais comme une tentative de contrôle stratégique d'un certain récit du républicanisme. Parce que Rousseau imagine les républiques comme des lieux d'idées, c'est-à-dire comme des entités politiques dont l'existence dépend de convictions partagées par tous les citoyens, il en vient dans la Lettre à considérer le cosmopolitisme littéraire comme dangereux pour l'autonomie et le maintien de la pensée et des mœurs sans lesquelles la république s'effondre. À partir de là, ce ne sont plus seulement la circulation des biens matériels et le mouvement de l'argent et des corps qui pervertissent la république, mais aussi la façon dont celle-ci est perçue et racontée par les pays qui l'entourent. $C^{\prime}$ est pourquoi la question du théâtre, éminemment politique, agit à plusieurs niveaux dans la Lettre. L'enjeu n'est pas seulement de prévenir l'établissement d'un théâtre à Genève, mais aussi de faire échec à une certaine théâtralisation de Genève sur la scène européenne.

La défense d'une frontière «intérieure» apparaît donc comme le but premier, quoique non explicitement déclaré, de Rousseau ${ }^{7}$. Il s'intéresse

6 Sur cette devise, consulter entre autres : Jean Starobinski, «The Motto Vitam impendere vero and the question of Lying», dans Patrick Riley (éd.), The Cambridge Companion to Rousseau, Cambridge, Cambridge University Press, 2001, p. 365-396.

7 On peut penser ici à la définition qu'Étienne Balibar donne de ce concept dans un de ses essais : «Des frontières intérieures désignent en quelque sorte la limite non représentable de toute frontière, telle qu'elle serait vue du dedans de son tracé. Aussi cette expression fait-elle immédiatement surgir l'ensemble des apories classiques de l'intériorité et de l'extériorité. Dans le contexte d'une réflexion sur 
à la limite telle qu'elle est perçue du dedans, c'est-à-dire dans le sentiment partagé d'une appartenance des citoyens à une entité politique qui leur est propre. Mais cette perception est médiatisée : elle repose en partie sur ce que l'on dit de la république et du républicanisme à l'extérieur de ses frontières. Un peu comme Rousseau donnera les Confessions ou les Rêveries du promeneur solitaire pour pouvoir affirmer son moi contre l'image du solitaire misanthrope que les philosophes auront construite autour de lui, il faut que la république, afin de continuer à exister, puisse se réaffirmer par des récits, s'identifier à elle-même, se reconnaître dans une certaine autoreprésentation contrôlée. Le miroir déformant de l'article de l'Encyclopédie est déjà une mise en scène, donc représente pour les citoyens de Genève le danger d'un détachement, d'une médiation où l'on peut se perdre ${ }^{8}$.

\section{Qui peut parler des républiques?}

La stratégie critique adoptée par Rousseau à propos de l'article de l'Encyclopédie consacré à Genève repose en grande partie sur ce que l'on pourrait appeler l'argument du relativisme politico-moral et de l'exception. Au nom d'une rigueur méthodologique qui veut qu'on n'applique pas les mêmes critères à des objets hétérogènes, Rousseau s'oppose en effet dès les premières pages à l'éloge que fait d'Alembert des pasteurs de Genève, puisque celui-ci les décrit presque comme des philosophes :

Pour tout dire en un mot, plusieurs pasteurs de Genève n'ont d'autre religion qu'un socinianisme parfait, rejetant tout ce qu'on appelle mystères, et

l'identité d'un peuple, d'une nation ou plus généralement d'un groupe humain, elle renvoie nécessairement à une problématique de la pureté, ou mieux de la purification, c'est-à-dire qu'elle pointe vers l'incertitude de cette identité : ce en quoi l'intérieur peut être pénétré ou adultéré par son rapport avec l'extérieur, qu'on appellera ici l'étranger, ou tout simplement pensé sans communication avec lui.» (Étienne Balibar, «Fichte et la frontière intérieure. À propos des Discours à la nation allemande», dans La Crainte des masses. Politique et philosophie avant et après Marx, Paris, Galilée, coll. «La Philosophie en effet», 1997, p. 133).

8 Au risque d'un anachronisme, il serait tentant de reconnaître une certaine parenté entre la Lettre et les réactions des députés de l'Assemblée lorsqu'il s'agira de défendre l'image et le récit de la Révolution française face aux nations et peuples $\mathrm{d}$ 'Europe, notamment au moment du jugement du roi. Cf. Robert Walzer, Régicide et Révolution. Le procès de Louis XVI. Discours et controverses, A Kupiec (trad.), Paris, Payot, collection «Critique de la politique», 1989. 
s'imaginant que le premier principe d'une religion véritable, est de ne rien proposer à croire qui heurte la raison : aussi quand on les presse sur la nécessité de la révélation, ce dogme si essentiel du Christianisme, plusieurs y substituent le terme d'utilité, qui leur parait plus doux : en cela s'ils ne sont pas orthodoxes, ils sont au moins conséquents à leurs principes ${ }^{9}$.

Effrayé de ces affirmations qui en d'autres contextes seraient à comprendre comme des accusations, Rousseau reproche au savant de ne pas avoir jugé les pasteurs genevois selon leurs critères propres, mais selon des vues sans rapports avec leurs véritables convictions : "Mais, Monsieur, quand on veut honorer les gens, il faut que ce soit à leur manière, et non pas à la nôtre ; de peur qu'ils ne s'offensent avec raison des louanges nuisibles, qui, pour être données à bonne intention, n'en blessent pas moins l'état, l'intérêt, les opinions, ou les préjugés de ceux qui en sont $1^{\prime}$ objet $^{10} . »$ Autrement dit, pour parler à bon escient de la république de Genève et de sa religion, il faudrait en faire partie, ou du moins pouvoir penser à la manière d'un citoyen. Parce qu'il est parisien, sujet d'une monarchie, parce qu'il est philosophe et Encyclopédistes, d'Alembert ne peut se prononcer sur Genève - il est doublement en décalage par rapport à son sujet.

Décrire les pasteurs genevois en déistes ou sociniens qui subordonneraient la religion à la philosophie, c'est pour Rousseau faire rentrer de force Genève dans l'Encyclopédie. D'ailleurs, si le protestantisme a mauvaise presse dans quelques milieux catholiques de la France du dix-huitième siècle, c'est aussi en partie parce qu'il est associé, au moins par certains, à la liberté de pensée et à l'ouverture à ce que l'on appelle l'esprit républicain. Jean Novi de Caveirac, dans son intolérante Apologie de Louis XIV publiée en 1758 (la même année que la Lettre), écrit sur le sujet du retour possible en France des Protestants exilés au moment de

9 Article «Genève» in Encyclopédie, ou dictionnaire raisonné des sciences, des arts et des métiers, Denis Diderot et Jean le Rond D'Alembert (éd.). University of Chicago : ARTFL Encyclopédie Projet (Winter 2008 Édition), Robert Morrissey (éd.), $<$ http://encyclopedie.uchicago.edu/>. (Orthographe modernisée par moi.)

10 Rousseau, Lettre à d'Alembert, p. 90. Rousseau, par une prudence toute philosophique, se montre toujours méfiant quant à la possibilité de pouvoir juger de ce qui est extérieur à soi : «Quand nous nous mettons à la place des autres nous nous y mettons toujours tels que nous sommes modifiés, non tels qu'ils doivent l'être, et quand nous pensons les juger sur la raison, nous ne faisons que comparer leurs préjugés aux nôtres.» (Jean-Jacques Rousseau, Essai sur l'origine des langues in CEuvres complètes, Paris, Gallimard, collection «Bibliothèque de la Pléiade», 1962, vol. V, p. 409). 
la Révocation des lignes qui résument à elles seules les enjeux posés de ce point de vue par l'éloge de d'Alembert : «Eh! que nous apporteraient [les Protestants], un esprit républicain, des erreurs sociniennes, des recrues pour nos frondeurs, des écoliers pour nos déistes ${ }^{11}$ ?» Même s'il ne s'agit pas ici d'aller jusqu'à dire que cette vision du protestantisme comme foyer de la libre pensée soit dominante au milieu du dixhuitième siècle, ni même fondée, on comprend mieux à la lumière d'un tel passage que l'éloge de Genève dans l'Encyclopédie puisse être reçu par Rousseau comme un cadeau empoisonné non seulement du point de vue de sa réception genevoise, mais aussi de sa réception européenne. La faute de d'Alembert est qu'il instrumentalise les pasteurs genevois pour les faire servir dans un débat qui leur est extérieur - la guerre qui oppose les philosophes et l'Église. En exhibant ainsi ces pasteurs sur la scène européenne et en faisant d'eux des crypto-philosophes, d'Alembert montre que ce n'est pas vraiment la ville de Genève qui l'intéresse, mais plutôt ce qu'elle peut servir à dire en France lorsqu'elle est ainsi violemment «déterritorialisée» par le discours philosophique de l'Encyclopédie.

Dans un développement qui fait pendant aux objections véhémentes qu'il adresse à d'Alembert, Rousseau fait valoir les titres qui lui permettent au contraire de parler de Genève avec autorité et légitimité, et de reprendre ainsi le contrôle de ce qui se dit publiquement sur Genève et le républicanisme. Deux idées peuvent de ce point de vue être dégagées à partir de la préface, qui servent à expliquer et justifier le bienfondé de l'intervention que représente la Lettre. La première est qu'il y va pour Rousseau d'une responsabilité de parole, celle d'un citoyen qui doit dire ce qu'il pense pour le bien de sa patrie : «[...] ne dois-je pas agir, parler, selon ma conscience et mes lumières? Ai-je dû me taire? L'ai-je $\mathrm{pu}$, sans trahir mon devoir et ma patrie ${ }^{12}$ ?» La deuxième justification tient au fait que Rousseau a écrit pour le camp des philosophes, qu'il a participé à l'Encyclopédie, donc qu'il est un personnage public dont l'opinion ne peut passer inaperçue. Sa réserve ferait immanquablement de lui le cosignataire de l'article «Genève»: «Pour avoir droit de

11 Jean Novi de Caveirac, Apologie de Louis XIV et de son conseil, sur la révocation de l'Édit de Nantes, pour servir de réponse à la Lettre d'un patriote sur la tolérance civile des Protestants de France, avec une dissertation sur la journée de la Saint-Barthélemy, 1758, p. 355. Pour une discussion approfondie des débats politiques au sein de Genève aussi bien qu'à son propos, voir ce texte important : Helena Rosenblatt, Rousseau and Geneva. From the First discourse to the Social Contract, 1749-1762, Cambridge, Cambridge University Press, 1997. 
garder le silence en cette occasion, il faudrait que je n'eusse jamais pris la plume sur des sujets moins nécessaires [...] ; il faudrait qu'on ignorât que j'ai eu quelques liaisons avec les éditeurs de l'Encyclopédie, que j'ai fourni quelques articles à l'ouvrage, que mon nom se trouve avec ceux des auteurs ; il faudrait que mon zèle pour mon pays fût moins connu, qu'on supposât que l'article "Genève" m'eût échappé, ou qu'on ne pût inférer de mon silence que j'adhère à ce qu'il contient. Rien de tout cela ne pouvait être, il faut donc parler $[\ldots]^{13} . »$ Ainsi Rousseau se pose à l'orée du texte à la fois en citoyen de Genève et en penseur européen, double appartenance qui lui confère précisément l'autorité adéquate pour traiter de la question du théâtre à Genève. Ne parle pas des républiques qui veut : c'est l'idée fondamentale qui dès le départ sous-tend la Lettre. Surtout, c'est encore une fois la question de l'autorité d'un discours, de sa signature, qui est posée.

Parallèlement à l'opposition déjà établie entre l'Encyclopédiste $\mathrm{d}^{\prime}$ Alembert et le citoyen Rousseau, il est possible d'en dégager une autre, qui cette fois prend place entre les lecteurs de l'Encyclopédie et le public auquel la Lettre est adressée - comme si à la question de l'autorité du texte, il fallait faire correspondre celle de ses destinataires. Rousseau prend en effet soin d'identifier ses lecteurs possibles : «[...] quoique je m'adresse à vous, j'écris pour le peuple $[\ldots]^{14} . »$ Comme des critiques l'ont déjà remarqué, cette phrase reflète une certaine complexité rhétorique inhérente au texte, liée au fait que celui-ci ne vise pas un public homogène ${ }^{15}$. Le «vous» employé ici par Rousseau est particulièrement ambigu : à un premier niveau il désigne bien sûr d'Alembert, mais à travers lui et par extension c'est sans doute l'ensemble des lecteurs de la France philosophique et de

13 Jean-Jacques Rousseau, Lettre à d'Alembert, p. 5-6.

14 Jean-Jacques Rousseau, Lettre à d'Alembert, p. 91-92.

15 Par ailleurs, il faut signaler avec Mostefai l'ambiguïté inhérente au choix du genre de la lettre, qui induit un certain flou quant à la question de la publicité du texte et de son positionnement rhétorique : «[...] si la Lettre est ostensiblement adressée à d'Alembert, et se présente comme une missive ouverte et publique, elle s'apparente tout aussi bien au genre de la lettre-pamphlet qu'à celui de la correspondance. Car le message qu'elle communique est à la fois d'ordre public et d'ordre privé, et on voit Rousseau osciller constamment entre ces deux modes qu'il maintient conjointement. S'adressant à d'Alembert, aux hommes de lettres européens, et au public genevois dans une sorte d'oraison publique, il tient également un discours plus personnel, secret, relégué en note, dans les marges, et sous le voile du latin. Texte public donc, mais aussi ésotérique et privé.» (Mostefai, Le Citoyen de Genève et la république des lettres, op. cit., p. 7). 
l'Europe pensante qui est visé. Quant à ce «peuple», à ces lecteurs «moyens» non rompus aux subtilités philosophiques qui, de l'aveu de Rousseau, l'obligent à ralentir le rythme de son texte, à ajouter exemples et détails, ce sont en premier lieu des citoyens de Genève, que Rousseau doit aussi convaincre de ne pas laisser s'installer un théâtre dans la république. Au moyen de ce décalage subtil entre celui ou ceux auxquels on s'adresse et ceux pour qui on écrit, Rousseau fait d'une pierre deux coups : il décrit Genève pour le reste de l'Europe, mais son véritable destinataire est le peuple de Genève. Rousseau, malgré ses protestations du contraire, maîtrise parfaitement sa rhétorique : si le citoyen ne peut parler de Genève qu'en affirmant qu'il s'adresse au peuple de Genève, c'est parce que parler de Genève directement au reste de l'Europe, simplement en répondant à d'Alembert, serait éminemment antipatriotique.

Ce que les nombreux commentateurs de ce texte n'ont peut-être pas assez souligné, et que l'on voit à l'œuvre ici, est que pour Rousseau la république de Genève ne peut s'écrire et se dire authentiquement que de l'intérieur et pour l'intérieur - dès qu'elle n'est plus parfaitement présente à elle-même, que l'on parle d'elle sans elle, en dehors d'elle, Genève se perd. En voulant contrôler ainsi ce qui se dit sur la république, le philosophe montre bien à quel point il la traite en texte, et en cela comme objet susceptible d'adultérations ruineuses. On serait même tenté de dire qu'il a ici pour sa patrie, pour Genève, la réaction qu'il aura plus tard à propos de ses propres œuvres, lorsqu'il imaginera la possibilité qu'elles soient transformées, après sa mort, sous la plume d'éditeurs hostiles : ici et là se reconnaît peut-être une même angoisse face à l'instabilité de tout récit, qu'il s'agisse de celui d'un citoyen ou de celui d'une république.

\section{La république et le rêve d'invisibilité}

L'article a rendu Genève trop présente, trop visible. Rousseau ne reproche pas tant à d'Alembert sa proposition d'installation d'un théâtre, que le fait qu'il ait donné la république en spectacle au reste de l'Europe : le théâtre n'est pas à sa place à Genève, mais Genève l'est encore moins dans l'Encyclopédie, c'est-à-dire sur cette "scène» d'autant plus ouverte qu'elle est virtuelle. Cette idée vient à l'explicite dans au moins deux passages. Le premier, au début du texte, est celui où Rousseau, reprenant d'Alembert sur son portrait des pasteurs genevois, choisit une expression qui métaphorise clairement la question du théâtre : «Plusieurs pasteurs de Genève n'ont, selon vous, qu'un socinianisme parfait. Voilà ce que vous déclarez à la face de 
$l^{\prime} E_{\text {Erope }}{ }^{16}$.» La déclaration n'est pas ici très loin de la déclamation, comme si Rousseau voyait en d'Alembert un acteur ou un orateur tourné vers un public. Plus que la réception genevoise de l'article, $c^{\prime}$ est sa réception européenne qui le préoccupe ici, c'est l'opinion publique à l'extérieur de Genève qui constitue le vecteur d'instabilité le plus fort : il y a là le danger d'une dissémination, le public n'étant ni connu, ni limité. Le second passage - qui marque un tournant dans la structure du texte — correspond au moment où Rousseau se justifie d'avoir jusque-là plus parlé du théâtre que de Genève : «Si je suis resté si longtemps dans les termes de la proposition générale, ce n'est pas que je n'eusse eu plus d'avantage encore à l'appliquer précisément à la ville de Genève, mais la répugnance de mettre mes concitoyens sur la scène m'a fait différer autant que je l'ai pu de parler de nous ${ }^{17}$.» L'emploi métaphorique du mot «scène» $\mathrm{n}$ 'a ici rien de fortuit : le texte devient scène, mise en scène, il montre d'ailleurs toujours trop, alors que Genève, elle, est pudique, réservée, modeste telle ces femmes que Rousseau décrit en gardiennes des mœurs et donc de la patrie. En suivant le mouvement de la Lettre, il faudrait considérer la Genève de l'article de l'Encyclopédie comme une sorte de ville travestie, fardée à l'image de Paris, à laquelle il faudrait opposer la Genève véritable, celle de la Lettre, une Genève qui ne se montre qu'à regrets sur cette scène européenne, uniquement lorsque l'on ne peut faire autrement. Cette opposition entre une Genève cachée, discrète, pudique, et un Paris qu'on pourrait, sans trop forcer les choses, qualifier d'obscène, permet de considérer les passages consacrés à la fête républicaine sous un nouveau jour ${ }^{18}$. Notamment, il faudrait distinguer deux niveaux dans le rapport entre communauté et visibilité.

Le premier niveau, le plus évident, correspond au groupe rassemblé qui se rend visible à lui-même. Lorsque Rousseau écrit plus loin qu'il faut donner «les spectateurs en spectacle ${ }^{19} »$, il s'agit pour lui de signifier les effets destructeurs d'une position d'extériorité par rapport au spectacle : dans le prolongement d'une métaphorisation du spectacle en communauté politique, il prône l'annulation de la scène, l'absence de parterre ou de loges d'où justement regarder et juger, afin que les spectateurs puissent devenir acteurs, c'est-à-dire citoyens actifs et non

16 Jean-Jacques Rousseau, Lettre à d'Alembert, p. 10 (c'est moi qui souligne).

17 Jean-Jacques Rousseau, Lettre à d'Alembert, p. 84.

18 Sur la question générale de la pudeur dans la Lettre, consulter : Coleman, Rousseau's political Imagination, op. cit., p. 113-118.

19 Jean-Jacques Rousseau, Lettre à d'Alembert, p. 115. 
pas sujets passifs. Rousseau donne ainsi des raisons précises pour expliquer que les fêtes sont les seules formes de spectacles véritablement républicaines : l'émulation, le renforcement des liens citoyens, la rencontre des hommes et des femmes sous le regard de tous, etc. L'inclusion se fait dans la simultanéité réciproque des regards : Jean Starobinski et Jacques Derrida ont bien montré que l'idéal républicain, l'authenticité des rapports interhumains, passe chez Rousseau par cette métaphore de la transparence ${ }^{20}$.

Mais à cela il faut ajouter un deuxième niveau. Car le salut de la république repose aussi sur la possibilité d'une certaine opacité : la fête doit être invisible à quiconque se place à l'extérieur. La république se donne en spectacle, certes, mais elle se donne en spectacle à elle-même et pour elle-même - l'assemblée républicaine est un cercle fermé. Les bals, tels que Rousseau les imagine, «ressembleraient moins à un spectacle public qu'à l'assemblée d'une grande famille ${ }^{21} \gg$ : il faut donc que ce que l'on croyait jusque-là public soit en fait du domaine du privé. Le lien entre les citoyens est si fort que leur rassemblement en devient exclusif, avec pour conséquence que tout regard extérieur est perçu comme une menace. La critique des salles de théâtre devrait donc être replacée au sein d'une seconde dialectique du visible et de l'invisible, de l'inclusif et de l'exclusif, qui agirait cette fois à l'échelle interétatique : «[...] n'adoptons point ces spectacles exclusifs qui renferment tristement un petit nombre de gens dans un antre obscur [...]. Non peuples heureux, ce ne sont pas là vos fêtes! c'est en plein air, c'est sous le ciel qu'il faut vous rassembler et vous livrer au doux sentiment de votre bonheur ${ }^{22}$.» La fête apparaît ici ouverte, mais il ne faut pas s'y tromper : toute la métaphore de la visibilité, de la transparence, qui détermine l'espace républicain en opposition avec l'obscurité et le renfermement monarchique des salles de théatre, n'a cours que si on se place à l'intérieur de la république. De l'extérieur, de Paris par exemple, celle-ci doit rester opaque et close. Rousseau redoute ainsi que l'établissement d'un théâtre, avec ses spectacles tardifs, obligerait les Genevois à «laisser une place forte ouverte pendant la nuit, au milieu de trois puissances dont la plus éloignée n'a pas une demi-lieue à faire pour arriver sur [leurs]

20 Jean Starobinski, Jean-Jacques Rousseau : la transparence et l'obstacle, Paris, Gallimard, 1971. Jacques Derrida, De la grammatologie, Paris, Minuit, 1967.

21 Jean-Jacques Rousseau, Lettre à d'Alembert, p. 120. Jean-Jacques Rousseau, Lettre à d'Alembert, p. 114. 
glacis $^{23}$.» Plus qu'une véritable préoccupation sur la capacité de Genève à assurer sa défense, il s'agit là d'un fantasme - l'ouverture du théâtre, c'est-à-dire la remise en question d'une frontière intérieure, entraînerait l'ouverture des portes et donc une violation de l'invisible dont la portée symbolique équivaudrait en violence à celle d'un affrontement armé.

De même, lorsque Rousseau évoque la présence d'étrangers venant assister aux fêtes et aux bals républicains, c'est pour presque immédiatement faire de ce regard extérieur la source d'une corruption. Certes, il y a d'abord la description d'une ouverture : «Sur ces idées, il serait aisé d'établir à peu de frais et sans danger, plus de spectacles qu'il n'en faudrait pour rendre le séjour de notre ville agréable et riant, même aux étrangers, qui, ne trouvant rien de pareil ailleurs, y viendraient au moins pour voir une chose unique ${ }^{24}$.» Ce passage, d'ailleurs, rappelle la dédicace du second discours, écrite cinq ans plus tôt, lorsque Rousseau oppose le luxe des palais parisiens, la pompe des spectacles, à l'ethos genevois : "À Genève, on ne trouvera que des hommes, mais pourtant un tel spectacle a bien son prix, et ceux qui le rechercheront vaudront bien les admirateurs du reste ${ }^{25} . »$ Mais, dans la Lettre, les choses ont changé, et cette ouverture au regard extérieur est annulée à l'instant même où elle est formulée : "Quoiqu'à dire le vrai, sur beaucoup de fortes raisons, je regarde ce concours comme un inconvénient bien plus que comme un avantage ; et je suis persuadé, quant à moi, que jamais étranger n'entra dans Genève qu'il n'y ait fait plus de mal que de bien $^{26}$.» Encore une fois, on note que la peur de la contagion, pendant traditionnel du républicanisme, n'est pas ici décrite de manière classique : ce n'est pas exactement la présence de l'étranger qui est mise en cause comme force corruptrice, mais la présence de l'étranger comme spectateur d'une fête qui incarne l'idéal républicain. C'est à nouveau de visibilité et d'invisibilité qu'il s'agit ici, d'autant que le spectateur étranger se transforme en trouble-fête parce qu'il ne sait pas voir. Dans la dernière note de la Lettre, passage très commenté, Rousseau décrit une fête genevoise spontanée dont il avait été témoin dans son enfance et ajoute : «Je sens bien que ce spectacle dont je fus si touché, serait sans attrait pour mille autres. Il faut des yeux faits pour le voir, et un cœur

23 Jean-Jacques Rousseau, Lettre à d'Alembert, p. 88.

24 Jean-Jacques Rousseau, Lettre à d'Alembert, p. 120.

25 Jean-Jacques Rousseau, Discours sur l'origine de l'inégalité in CEuvres complètes, Paris, Gallimard, collection «Bibliothèque de la Pléiade», 1964, vol. III, p. 120.

Ibid. 
fait pour le sentir ${ }^{27} . »$ Encore une fois — bien que cela mériterait d'être illustré par d'autres exemples empruntés à d'autres textes - le républicanisme est pour Rousseau un phénomène fermé ${ }^{28}$.

\section{Conclusion}

Pour finir, il faut souligner le fait que Rousseau, dans son acte d'écriture citoyenne et patriotique, se trouve aux prises avec une sorte d'aporie : si le républicanisme, pour continuer à exister, doit se rendre invisible au reste de l'Europe, alors la Lettre ne fait-elle pas en effet infraction au principe qu'elle expose? Signe de sa finesse et de son sûr instinct de polémiste, Rousseau semble parfaitement conscient de l'ambiguité de sa propre intervention, et c'est de toute évidence pour répondre à cette objection qu'il prend soin de décrire sa prise de parole comme quelque chose d'exceptionnel, qui ne sera jamais répété : il le fait d'abord dans la préface, où il présente sa Lettre comme son écrit ultime («Lecteur, si vous recevez ce dernier ouvrage avec indulgence, vous accueillerez mon ombre ; car pour moi je ne suis plus $\left.{ }^{29} \gg\right)$, puis dans les dernières lignes du texte, où il reprend à peu de choses près la même idée («Puisse [la jeunesse] transmettre à ses descendants les vertus, la liberté, la paix qu'elle tient de ses pères! C'est le dernier vœu par lequel je finis mes écrits ; c'est celui par lequel finira ma vie $\left.{ }^{30} . »\right)$ Tout se passe comme si Rousseau ne pouvait écrire le républicanisme que d'outre-tombe, comme si l'acte d'avoir fait monter sur scène ses concitoyens, d'avoir parlé d'eux et en leur nom devant le public parisien, acte si contraire au républicanisme, ne pouvait s'envisager que dans le sacrifice de soi. Ce serait pour compenser l'acte de dévoilement un peu sacrilège de la Lettre que Rousseau aurait choisi de donner son texte tout en s'effaçant symboliquement — un peu comme le législateur, tel Lycurgue à Sparte,

Jean-Jacques Rousseau, Lettre à d'Alembert, p. 123.

28 Le moment des Confessions où Rousseau décrit son départ de Genève après en avoir trouvé les portes fermées mériterait d'être relu sous cet angle : au tout début de la suite d'événements qui mènera Rousseau à l'écriture se trouve une expérience d'extériorité par rapport à la république. À partir de là, Rousseau ne sera plus un citoyen comme les autres, puisqu'il ne sera pas intérieur à la république mais dans une sorte d'entre-deux de l'exil qui rendra possible et même nécessaire l'écriture.

29 Jean-Jacques Rousseau, Lettre à d'Alembert, p. 7.

30 Jean-Jacques Rousseau, Lettre à d'Alembert, p. 125. 


\section{Lucien Nouis}

disparaît une fois qu'il a donné les lois à son peuple, parce qu'il doit rester extérieur à ce qu'il fonde. Bien sûr, Rousseau ne s'arrête pas d'écrire, et cette mise en scène de l'intervention citoyenne est douteuse. Elle n'en reste cependant pas moins révélatrice de la façon dont Rousseau imagine d'éviter à Genève le sort des républiques de l'antiquité. La stratégie de la Lettre serait de remédier à la mise en spectacle dangereuse effectuée par l'article de d'Alembert en ouvrant justement aux regards, dans un geste paradoxalement antirépublicain, la cité républicaine - dévoilement qui n'aurait d'autre but que de convaincre le reste de l'Europe de laisser Genève, oubliée et heureuse, se retrancher dans son âge d'or. Prises dans ce sens, les contradictions de la lettre apparaissent moins mystérieuses : il faut à la fois inscrire et désinscrire le républicanisme genevois pour qu'il continue d'exister, c'est-à-dire le mettre une dernière fois en scène pour expliquer au monde la nécessité de n'en plus parler.

\section{LUCIEN NOUIS}

New York University 